Pacific Journal of Mathematics

RIGHT CHAIN RINGS AND THE GENERALIZED SEMIGROU 


\title{
RIGHT CHAIN RINGS AND THE GENERALIZED SEMIGROUP OF DIVISIBILITY
}

\author{
H. H. BRUngs AND G. TÖRNER
}

Let $R$ be a ring with unit element and without zerodivisors and let $\tilde{H}(R)=\{\tilde{x} \mid 0 \neq x \in R\}$ where $\tilde{x}$ is the mapping from the set of all nonzero principal right ideals of $R$ into itself defined by $\tilde{x}(a R)=x a R$. $\tilde{H}(R)$ is a partially ordered semigroup that can be considered as a generalization of the group of divisibility of a commutative integral domain. We study those rings $R$ for which $\widetilde{H}(R)$ is totally ordered.

1. Introduction. Associated with any commutative integral domain $A$ is the partially ordered group $G(A)$ of nonzero fractional principal ideals of $A$ with $a A \leqq b A$ if and only if $a A$ contains $b A$. It is well known (see [4], [5], [8]) that $G(A)$, the group of divisibility, reflects certain properties of $A$, like $A$ being a unique factorization domain, the fact that any two elements in $A$ have a greatest common divisor or $A$ being a valuation ring. This concept of a group of divisibility cannot be extended directly to a not necessarily commutative integral domain $R$.

In this paper we associate with any ring $R$ with unit element and without zero-divisors a partially ordered semigroup $\tilde{H}(R)$ which is isomorphic to the semigroup $H(A) \subseteq G(A)$ of nonzero principal ideals $a A$ in $A$ if $A$ is a commutative domain.

After observing some basic facts about $\widetilde{H}(R)$ we characterize in $\S 3$ those rings $R$ with $\widetilde{H}(R)$ totally ordered as right chain rings $R$ with $J a \subseteq a R$ for all $a$ in $R$ and $J=J(R)$ the Jacobson radical of $R$. These rings are localizations of right invariant right chain rings. The main result of $\S 4$ is the theorem that a ring with $\tilde{H}(R)$ totally ordered and d.c.c. for prime ideals is right invariant. In a final $\S 5$ we show by examples that for every totally ordered group $G$ there exists a ring $R$ with $\widetilde{H}(R)$ totally ordered and $G$ (not only the positive cone of $G$ ) can be embedded into $\widetilde{H}(R)$. The value group $G(A)$ is particularly useful in case $A$ is a commutative valuation ring. The nonzero principal right ideals in a right chain ring $R$ form a semigroup $H(R)$ under ideal multiplication only if $R$ is right invariant. In the general case it is the semigroup $\tilde{H}(R)$ which takes the place of $H(R)$. Mathiak in [6] studies right and left chain domains with the help of a group that could be considered a generalization of $G(A)$. We found that in the case of one-sided conditions a generalization of $H(A)$, which will be a semigroup only, will be more natural.

2. Definition and preliminary results. We consider only rings 
with unit element and without zero-divisors. We call a ring $R$ right invariant if $R a \subseteq a R$ (if and only if $R a R=a R$ ) holds for all elements $a$ in $R$ and $R$ is a right chain ring (sometimes called a right valuation ring) if for $a, b$ in $R$ either $a R \subseteq b R$ or $b R \subseteq a R$ holds. Here $I \subset L$ always means that the set $I \neq L$ is contained in $L ; J=$ $J(R)$ is the Jacobson radical and $U=U(R)$ the group of units of $R$.

Let $W=\{a R \mid 0 \neq a$ in $R\}$ be the set of nonzero principal right ideals of $R$. Every element $0 \neq x$ in $R$ induces a mapping $\widetilde{x}$ on $W$ with $\widetilde{x}(a R)=x a R$; and $\widetilde{x y}=\widetilde{x} \widetilde{y}$ follows. With $\widetilde{x} \geqq \widetilde{y}$ defined as $x a R \leqq y a R$ for all $a$ in $R$ we can consider $\tilde{H}(R)=\{\widetilde{x} \mid 0 \neq x$ in $R\}$ as a partially ordered semigroup. Further, $\widetilde{x+y} \geqq \inf (\widetilde{x}, \widetilde{y})$; i.e., $\widetilde{z} \leqq \widetilde{x}$, $\widetilde{z} \leqq \widetilde{y}$ implies $\widetilde{z} \leqq \widetilde{x+y}$. The mapping ' $\sim$ ' from $R^{*}(=R \backslash 0)$ to $\widetilde{H}(R)$ is called the regular right valuation of $R$ with the value-semigroup $\widetilde{H}(R)$. This semigroup satisfies the following conditions:

(1) $\widetilde{H}(R)$ is a partially ordered semigroup with unit element $\tilde{1}$.

(2) $\widetilde{x} \leqq \widetilde{y}$ if and only if there exists a $\widetilde{t}$ in $\widetilde{H}(R)$ with $\widetilde{x} \widetilde{t}=\widetilde{y}$ and $\widetilde{1} \leqq \widetilde{t}$.

(3) $\tilde{x} \widetilde{y}=\widetilde{x} \widetilde{z}$ implies $\widetilde{y}=\widetilde{z}$ for $\widetilde{x}, \widetilde{y}, \widetilde{z}$ in $\widetilde{H}(R)$.

This means that the order in $\tilde{H}$ is a right natural order and $H$ is left cancellative.

We draw a few immediate conclusions from these properties:

(i) $\widetilde{x} \leqq \tilde{1}$ implies that $\widetilde{x}$ is a unit in $\widetilde{H}$, i.e., there exists $\widetilde{y}$ with $\widetilde{x} \widetilde{y}=\widetilde{y} \widetilde{x}=\widetilde{1}$.

(ii) $\tilde{1} \leqq \widetilde{x}$ implies $\widetilde{x} \widetilde{a}=\widetilde{a} \widetilde{x}^{\prime}$ for some $\widetilde{x}^{\prime}$ in $\widetilde{H}$.

To prove (i) we have by (2) an element $\tilde{t}$ with $\tilde{x} \tilde{t}=\tilde{1}$. This implies $\widetilde{x} \tilde{t} \widetilde{x}=\widetilde{x}$ and $\widetilde{t} \widetilde{x}=\widetilde{1}$ using (3). For $\tilde{1} \leqq \widetilde{x}$ and $\widetilde{a}$ in $\widetilde{H}$ we have $\widetilde{a} \leqq \widetilde{x} \widetilde{a}$ and $\widetilde{x} \widetilde{a}=\widetilde{a} \widetilde{x}^{\prime}$ for some $\widetilde{x}^{\prime}$ using (2) again. Let $\widetilde{U}=\widetilde{U}(R)$ be the subgroup of units of $\widetilde{H}(R)$. The following condition is satisfied by $\widetilde{H}(R)$ :

(4) Let $\widetilde{U}^{\prime}$ be a subgroup of $\widetilde{U}$ with $\widetilde{U}^{\prime} \widetilde{x} \subseteq \widetilde{x} \widetilde{U}$ for all $\widetilde{x}$ in $\tilde{H}(R)$. Then $\widetilde{U}^{\prime}=\{\tilde{1}\}$. In particular $\widetilde{U}=\{\tilde{1}\}$ for $R$ commutative. The following is an easy example of a semigroup $S$ satisfying conditions (1)-(3), but not (4).

Let $S=\{(n, a) ; n, a \in Z ; n \geqq 0\}$ considered as a subsemigroup of $G=\boldsymbol{Z} \oplus \boldsymbol{Z} ; \boldsymbol{Z}$ the integers. We write $(n, a)>(m, b)$ if either $n>m$ or $n=m$ and $a>b$. Conditions (1), (2), (3) hold for $S$, but $U=$ $\{(0, a) ; a \in Z\}$ is a subgroup $\neq\{e\}$ of $S$, violating (4).

Two obvious problems arise: What is the structure of semigroups with (1), (2), (3), (4)? Given a semigroup $S$ satisfying (1), (2), (3), (4) is $S \cong \widetilde{H}(R)$ for some $R$ ? We are not able to answer these questions in general.

Definition. Let $R$ be a ring. Then 


$$
\hat{R}=\{r \in R \mid \widetilde{r} \geqq 1\} \cup\{0\}=\{r \in R \mid r a R \leqq a R \text { for all } a \text { in } R\} .
$$

It is obvious that $\hat{R}$ is a subring of $R$.

LEMma 1. (1) $\hat{R} a \subseteq a \hat{R}$ for all a in $R$; in particular $\hat{R}$ is a right invariant subring of $R$.

(2) The mapping âी to $\tilde{a}$ for $a \neq 0$ in $R$ defines an isomorphism between the semigroup $C(R)$ of $\hat{R}$-modules a $\hat{R}$ with a in $R$ onto $\widetilde{H}(R)$. In $C(R)$ we have $a \hat{R} b \hat{R}=a b \hat{R}$ as operation and $a \hat{R} \leqq b \hat{R}$ if and only if $a \hat{R} \supseteqq b \hat{R}$.

(3) $\tilde{H}(R) \simeq R^{*} / U(\hat{R})$ where $U(\hat{R})$ is the group of units of $\hat{R}$ and $r_{1} \equiv r_{2}$ if and only if $r_{1}=r_{2} u$ with $u$ in $u(\hat{R})$ defines $a$ congruence relation on $R^{*}$, the multiplicative semigroup of nonzero elements in $R$.

Proof. (1) $\hat{R} a \leqq a R$ by definition. If $r$ is in $\hat{R}$ then $r a=a r_{1}$ and $r a b=a b r_{2}=a r_{1} b$ for any $a, b$ in $R$ with $r_{1}, r_{2}$ in $R$. But $r_{1} b=$ $b r_{2}$ implies $r_{1}$ in $\hat{R}$ and $\hat{R} a \subseteq a \hat{R}$ for $a \neq 0$ in $R$.

(2) Using (1) it follows that $a \hat{R} b \hat{R}=a b \hat{R}$ for $a, b$ in $R$. If $\widetilde{a} \geqq \widetilde{b}$ then $a x R \subseteq b x R$ for all $x$ in $R$ and $a=b s$ and $s$ in $\hat{R}$, hence $a \hat{R} \cong b \hat{R}$ follows. Reversing these arguments yields the converse and $\tilde{H}(R) \simeq\{a \hat{R} \mid 0 \neq a$ in $R\}$ as a partially ordered semigroup.

$(3)$ is just a different version of (2).

REMARK. If $R$ is embeddable into some skew field then $\hat{R}=$ $\bigcap_{0 \neq a \in R} a R a^{-1}$.

If $R$ is a ring such that the product of any two nonzero principal right ideals is again a nonzero principal right ideal we write $H(R)$ for the semigroup of the nonzero principal right ideals of $R ; H(R)$ is a partially ordered semigroup with $a R \geqq b R$ if and only if $a R \subseteq b R$.

If $H(R)$ exists and is isomorphic to $\tilde{H}(R)$ under the mapping that assigns $\tilde{x}$ to $x R$ then $R$ is right invariant. On the other hand $H(R)$ does exist for some rings that are not right invariant; simple rings or not right invariant principal ideal domains are obvious examples.

The following lemma shows that $H(R)$ exists for a local ring $R$ if and only if $R$ is right invariant.

Lemma 2. Assume $H(R)$ exists and let $0 \neq a$ be in $R$. Then $R a R=b R$ for some $b$ and if $a=b c$ then $c$ is not contained in $J(R)$.

Proof. It only remains to show that $c$ is not in $J(R)$. We have $b=\sum r_{i} a s_{i}$ for some $r_{i}, s_{i}$ in $R ; b=\sum r_{i} b c s_{i}=\sum b r_{i}^{\prime} c s_{i}=b \sum r_{i}^{\prime} c s_{i}$ where 
$r_{i} b=b r_{i}^{\prime}$ for some $r_{i}^{\prime}$ in $R$. But this is impossible for $c$ in $J(R)$.

Corollary. If $R$ is local then $H(R)$ exists if and only if $R$ is right invariant.

3. $\widetilde{H}(R)$ totally ordered. If $A$ is a commutative integral domain its group of divisibility $G(A)$ is totally ordered only if $A$ is a valuation ring. We will discuss the corresponding question for $\widetilde{H}(R)$ and characterize the rings with $\tilde{H}(R)$ totally ordered. If $x$ and $y$ are nonzero elements in $R$ then $\widetilde{x} \leqq \widetilde{y}$ or $\widetilde{y}<\widetilde{x}$ and $x R \supseteqq y R$ or $y R \supseteqq x R$ follows. Therefore, $R$ is a right chain ring if $\widetilde{H}(R)$ is totally ordered. Examples (see §5) show that for $R$ a right chain ring $\widetilde{H}(R)$ is not necessarily totally ordered.

TheOREM 1. For an integral domain $R$ the following conditions are equivalent:

(1) $\tilde{H}(R)$ is totally ordered.

(2) $R$ is a right chain ring such that $r$ in $R$, not in $\hat{R}$ implies $r^{-1}$ in $\hat{R}$.

(3) $R=R_{P}^{\prime}$, the localization of a right invariant right chain ring $R^{\prime}$ at a prime ideal $P$ of $R^{\prime}$.

(4) $R$ is a right chain ring such that $J a \subseteq a R$ for all a in $R$.

(5) $R$ is a right chain ring and if $R a \varsubsetneqq a R$ then $J a \subseteq a J$ for any $a$ in $R$.

(6) The submodules of the right $\hat{R}$-module $R$ are totally ordered.

Proof. (1) $\Rightarrow(2)$ We observed that $R$ is a right chain ring if $\widetilde{H}(R)$ is totally ordered. For an element $r$, not in $\hat{R}$, we have $\widetilde{r}<\widetilde{1}$, hence $r a R \supseteqq a R$ for all $a \in R$ and $r$ in $U(R), r^{-1}$ in $\hat{R}$ follows. $(2) \Rightarrow(3)$ It follows from (2) that $\hat{R}$ is a right chain ring and from Lemma 1 that $\hat{R}$ is right invariant. The set $S=\hat{R} \cap U(R)$ is multiplicatively closed and $P=\hat{R} \backslash S$ is a prime ideal in $\hat{R}$. Finally, $R=$ $\hat{R}_{P}=\hat{R} S^{-1}$ is the localization of $\hat{R}$ at $P$.

To prove that (3) implies (1) we need a few lemmas.

Let $R$ be a right invariant right chain ring. We write $\widetilde{T}=$ $\{\widetilde{t} \in \widetilde{H}(R) \mid t \in T\}$ for a subset $T \subseteq R^{*}$ and we say $\widetilde{T}(\neq \varnothing)$ is $R$-con$v e x$ if for $t R \cong s R \subseteq R, t$ in $T$, the element $\widetilde{s}$ is contained in $\widetilde{T}$. One can check the following two statements.

LEMMA 3. There is a one-to-one correspondence between the set of $R$-convex subsets of $\tilde{H}(R)$ and the right ideals $\neq R$ given by

$$
\begin{aligned}
\widetilde{S} \longrightarrow \widetilde{S}^{\prime} & =\{x \in R \mid \widetilde{x} \notin \widetilde{S}\} \cup\{0\} \\
I \longrightarrow I^{\prime} & =\{\widetilde{x} \in \widetilde{H}(R) \mid x R \supset I\}
\end{aligned}
$$


where $\widetilde{S}$ is $R$-convex and $I$ is a right ideal $\neq R$.

Lemma 4. The R-convex subset $\widetilde{S}$ is a subsemigroup of $\widetilde{H}(R)$ if and only if $\widetilde{S}^{\prime}=P$ is a completely prime ideal of $R$.

We consider the situation as described in the last lemma. Then $S=\{x \in R \mid \widetilde{x} \in \widetilde{S}\}$ is a multiplicatively closed saturated (i.e., $a b$ in $S$ implies $a, b$ in $S$ ) right Ore system in $R$. The corresponding prime ideal is $P=R \backslash S$ and $R_{P}=R S^{-1}$ is the corresponding localization. Set $N=N(S)=\left\{r \in R \mid r a=a s_{a}, s_{a}\right.$ in $S$ for all $a \neq 0$ in $\left.R\right\} . \quad N$ is an $R$-convex subsemigroup of $S$ maximal with the property that $a^{-1} N a \subseteq N$ for all nonzero $a$ in $R$. To see this, one observes that with $n$ in $N, n R \subseteq m R \subseteq R$, we have $n=m r$ for some $r$ and $n a=$ $a s_{a}=a m^{\prime} r^{\prime}$ for $m^{\prime}, r^{\prime}$ in $R$ with $m a=a m^{\prime}, r a=a r^{\prime}$. Therefore $m^{\prime} r^{\prime}=s_{a}$ is in $S$ and $m^{\prime}$ in $S$, and $m$ in $N$. Further, $n$ in $N$ and $n a=a s_{a}$ implies $s_{a}$ in $N$.

To $N$ there corresponds a prime ideal $Q=R \backslash N$ with $P \subseteq Q \subseteq J$. We want to describe $\widetilde{H}\left(R_{P}\right)$ and we will get the result by considering two special cases:

(i) $N(S)=S$, i.e., $Q=P$ (Lemma 5) and

(ii) $N(S)=U(R)$, i.e., $Q=J$ (Lemma 6).

LEMMA 5. Let $R$ be a right invariant right chain ring, $P$ a prime ideal in $R, S=R \backslash P$. Assume $N(S)=N=S$. Then $R_{P}$ is again right invariant and $\widetilde{H}\left(R_{P}\right) \simeq \widetilde{H}(R) / \widetilde{N}=\bar{H}$.

Proof. That $R_{P}$ is again right invariant follows from the fact that every principal right ideal in $R_{P}$ has the form $a R_{P}$ with $a$ in $R$ and that $s a=a s_{a}$ for all $a$ in $R, s_{a}$ in $S$ if $s$ is in $S=N$. Hence $r s^{-1} a R_{P}=r a R_{P}=a r^{\prime} R_{P}$ with $r a=a r^{\prime}, r, a$ in $R$. If one defines $\widetilde{r}_{1} \equiv \widetilde{r}_{2}, r_{1}, r_{2}$ nonzero elements in $R$, if and only if $r_{1}=r_{2} n$ or $r_{1} n=r_{2}$ for some $n$ in $N$, then " $\equiv$ " is a congruence relation defined on $\tilde{H}$, and we write $H=\widetilde{H}(R) \backslash \widetilde{N}$ for the factor semigroup modulo this congruence. Further, $\bar{r}_{1}>\bar{r}_{2}$ in $\bar{H}$ if and only if $r_{1}>r_{2}$ in $\widetilde{H}(R)$ and $\widetilde{r}_{1} \not \equiv \widetilde{r}_{2}$. It follows that $\bar{H} \simeq \widetilde{H}\left(R_{P}\right)$ as totally ordered semigroups.

LeMma 6. Let $R$ be a right invariant, right chain ring, $P$ a prime ideal in $R, S=R \backslash P$. Assume $N(S)=U(R)$. Then $R_{P}$ is not right invariant if $P \subset J$ and $\widetilde{H}\left(R_{P}\right) \simeq \widetilde{H}(R) \widetilde{S}^{-1}$.

Proof. $\tilde{H}(R)$ contains the subsemigroup $\widetilde{S}$. We will prove that under the above assumption $\widetilde{H}(R)$ can be embedded into the semigroup $\widetilde{H}(R) \widetilde{S}^{-1}=\left\{\widetilde{r} \widetilde{s}^{-1} \mid r \in R^{*}, s \in S\right\}$ of fractions for $\widetilde{H}(R)$. 
The semigroup $\tilde{H}(R)$ is totally ordered and $\alpha \beta=\alpha \gamma$ for $\alpha, \beta, \gamma$ in $\tilde{H}(R)$ implies $\beta=\gamma$. Since the other cancellation law does not hold in general, $\widetilde{H}(R)$ itself may not be embeddable into a group. But for every $\widetilde{r}$ in $\tilde{H}(R)$ and $\widetilde{s}$ in $\widetilde{S}$ there exists an element $\widetilde{a}$ in $\widetilde{H}(R)$ with $\widetilde{r} \widetilde{a}=\widetilde{s}$ or $\widetilde{r}=\widetilde{s} \widetilde{a}$ and $\tilde{H}(R) \widetilde{S}^{-1}$ exists ([3], Prop. 5.1; page 21 ) if we can show that $\widetilde{r}_{1} \widetilde{s}=\widetilde{r}_{2} \widetilde{s}$ implies $\widetilde{r}_{1}=\widetilde{r}_{2}$ for $\widetilde{r}_{1}, \widetilde{r}_{2}$ in $\widetilde{H}(R)$, $\widetilde{s}$ in $\widetilde{S}$.

We can assume $r_{1}=r_{2} c$ for some $c$ in $R$ and we are done if we can show that $c$ is in $N$. But, $\widetilde{r}_{1} \widetilde{s}=\widetilde{r}_{2} \widetilde{s}$ implies $r_{2} c s=r_{2} s \varepsilon$ for some $\varepsilon$ in $U(R)$. Therefore $c s=s \varepsilon$ and $c$ is an element of $S$. Let $a$ be in $R$. If $a$ is in $S$ then $c a=a c^{\prime}$ with $c^{\prime}$ in $S$. If $a$ is not in $S$ then $a=s a_{1}$ for some $a_{1}$ in $R$ and $c a=c s a_{1}=s \varepsilon a_{1}=s a_{1} \varepsilon^{\prime}=a \varepsilon^{\prime}$ with $\varepsilon^{\prime}$ in $U(R)$. Hence, $c$ is in $N=U(R)$ and $K=\widetilde{H}(R) \widetilde{S}^{-1}=\left\{\widetilde{r} \widetilde{s}^{-1} \mid r \in R^{*}, s \in S\right\}$ exists.

This semigroup is totally ordered if we define $\widetilde{r}_{1} \widetilde{s}_{1}^{-1} \geqq \widetilde{r}_{2} \widetilde{s}_{2}^{-1}$ if and only if for all $\widetilde{s}, \widetilde{s}^{\prime}$, with $\widetilde{s}_{1} \widetilde{s}=\widetilde{s}_{2} \widetilde{s}^{\prime}$ we get $\widetilde{r}_{1} \widetilde{s} \geqq \widetilde{r}_{2} \widetilde{s}^{\prime}$.

This last condition is equivalent to $\widetilde{r}_{1} \geqq \widetilde{r}_{2} \widetilde{s}$ if $s_{1}=s_{2} s$ and $\widetilde{r}_{1} \widetilde{s} \geqq \widetilde{r}_{2}$ if $s_{1} s=s_{2}$ where $s$ is some element in $S$. For the necessary computations it is the easiest to write any finite number of elements in $K$ in the form $\widetilde{r}_{i} \widetilde{s}^{-1}, i=1, \cdots, n$.

It is a bit tedious to check that $K$ is a totally ordered semigroup with unit element such that

(i) $\alpha \geqq \beta$ in $K$ implies that there exists $\gamma$ in $K$ with $\alpha=\beta \gamma$

(ii) $\gamma \alpha=\gamma \beta$ implies $\alpha=\beta$ where $\alpha, \beta, \gamma$ are in $K$.

Further, it follows from these conditions that all elements $\gamma \leqq \widetilde{1}$ in $K$ have an inverse in $K$.

It remains to show that $K \simeq \widetilde{H}\left(R_{P}\right)$ as ordered semigroups where the isomorphism is given by $\widetilde{r} \widetilde{s}^{-1} \leftrightarrow \widetilde{r s^{-1}}$. (Here $\widetilde{r}, \widetilde{s}$ are elements in $\widetilde{H}(R), \widetilde{r s}^{-1}$ is an element in $\widetilde{H}\left(R_{P}\right)$.) We shall show here that the given correspondence is one-to-one and omit the rest.

Let $\widetilde{r_{1} s^{-1}}=\widetilde{r_{2} s^{-1}}$ i.e., $r_{1} s^{-1} a R_{P}=r_{2} s^{-1} a R_{P}$ for all $a$ in $R_{P}$; in particular $r_{1} s^{-1} s b R_{P}=r_{2} s^{-1} s b R_{P}$ for all $b$ in $R$ and $r_{1} b R_{P}=r_{2} b R_{P}$, $r_{1} b=r_{2} b s^{\prime}$ or $r_{1} b s^{\prime}=r_{2} b$ for some $s^{\prime}$ in $S$ follows. Comparing $r_{1}$ and $r_{2}$ yields $r_{1}=r_{2} c$ or $r_{2}=r_{1} c$ for some $c$ in $N$ and $\widetilde{r}_{1}=\widetilde{r}_{2}$ in $\widetilde{H}(R)$. If conversely $\widetilde{r}_{1} \widetilde{s}^{-1}=\widetilde{r}_{2} \widetilde{s}^{-1}$ in $K$ we get $\widetilde{r}_{1}=\widetilde{r}_{2}$ in $\widetilde{H}(R)$ and therefore $r_{1} s^{-1} a R_{P}=r_{2} s^{-1} a R_{P}$ for all $a$ in $R$ : If $a$ is in $S$ this is obvious, otherwise $a=s b$ and $r_{1} b R=r_{2} b R$ implies $r_{1} s^{-1} a R_{P}=r_{2} s^{-1} a R_{P}$ in that case. Finally let $s$ be in $S \backslash U(R)$. Then there exists $a$ in $R$ with $s a=a g$ and $g$ not in $S$ since $s$ is not in $N$. This shows that $s^{-1} a R_{P} \supset a R_{P}$ and $R_{P}$ is not right invariant.

If we combine Lemma 5 and Lemma 6 we get the following result:

THEOREM 2. Let $R$ be a right invariant right chain ring, $P$ a 
prime ideal in $R, S=R \backslash P ; N=\left\{x \in R \mid x a=a s_{a}, s_{a}\right.$ in $S$ for all $a \in R\}$. Then:

(1) $\tilde{H}\left(R_{P}\right) \simeq \bar{H} \bar{S}^{-1}$ is a totally ordered semigroup with $\bar{H}=$ $\widetilde{H}\left(R_{Q}\right) \simeq \widetilde{H}(R) / \widetilde{N}$ and $\bar{S} \simeq \widetilde{S} / \widetilde{N} ; Q=R \backslash N$ is a prime ideal and $R_{Q}$ is right invariant.

(2) $R_{P}$ is right invariant if and only if $N=S$.

With Theorem 2 the equivalence of (1), (2), (3) in Theorem 1 is proven.

We prove the equivalence of (1) and (4). If $\widetilde{H}(R)$ is totally ordered and $j$ in $J(R)$, then $\tilde{j} \leqq \widetilde{1}$ is impossible, since this implies $j R=R, j$ a unit. Hence $j a R \subseteq a R$ for all $a$ in $R$. Conversely if $R$ is a right chain ring with $J a \subseteq a R$ for all $a$ in $R$ we must show that for any nonzero elements $x, y$ in $R$ either $\widetilde{x} \leqq \widetilde{y}$ or $\widetilde{y} \leqq \widetilde{x}$. If we assume on the contrary that there exist $a, b$ in $R$ with $x a R \subset$ $y a R$ and $y b R \subset x b R$ we obtain $x a=y a v_{1}, y b=x b v_{2}$ and say $a=b s$ for $v_{1}, v_{2}, s$ in $J$ (the case $b=a s$ is similar). Then $y a=y b s=$ $x b v_{2} s=x b s v_{2}^{\prime}=x a v_{2}^{\prime}=y a v_{1} v_{2}^{\prime}$ and $y a=0$ where $v_{2} s=s v_{2}^{\prime}$ for some $v_{2}^{\prime}$ in $R$, using (4).

The implication $(5) \Rightarrow(4)$ is obvious. To prove $(4) \Rightarrow(5)$ assume there is an $a$ in $R$ with $R a \nsubseteq a R$ and $J a \nsubseteq a J$, but $J a \subseteq a R$. Then there exist elements $u$ in $U(R), n$ in $J$ with $u a R \supset a R$ and $u a n=a$; and elements $n^{\prime}$ in $J, u^{\prime}$ in $U(R)$ with $n^{\prime} a$ in $a R$, but not in $a J$, hence $n^{\prime} a u^{\prime}=a$. This leads to $u n^{\prime} a u^{\prime} n=a$ and with $J a \leqq a R$ to $a=0$, a contradiction. The equivalence of (1) and (6) follows from Lemma $1(2)$ and with this Theorem 1 is proved completely.

Definition. A right chain ring $R$ that satisfies the equivalent conditions of Theorem 1 is called semi-invariant.

Since $\widetilde{H}(R)$ is not known even if $R$ is right invariant unless $R$ is also right noetherian or satisfies some other extra condition (see [1]) we cannot describe the structure of $\widetilde{H}(R)$ for a semi-invariant ring $R$. It follows from Theorem 2 that this semigroup is a group of fractions of a semigroup $H=\widetilde{H}\left(R^{\prime}\right)$ where $R^{\prime}$ is a right invariant right chain ring with respect to a subsemigroup $T$ of $H$ which satisfies

(1) If $t$ is in $T, h$ in $H$ and $e$ the unit element in $H$ with $e \leqq h \leqq t$, then $h$ is in $T$.

(2) For every $e \neq t$ in $T$ there exist $h$ and $k$ in $H$ with $t h=h k$ and $k$ not in $T$.

(3) $h_{1} t=h_{2} t$ for $t$ in $T, h_{1}, h_{2}$ in $H$ implies $h_{1}=h_{2}$.

One sees that $\tilde{H}(R), R$ semi-invariant, not a division ring, is not a group, but we will show that for every totally ordered group $G$ 
there exists a semi-invariant ring $R$ such that $G$ can be embedded into $\tilde{H}(R)$.

4. Semi-invariant right chain rings with d.c.c. for prime ideals. Investigating the condition $\tilde{H}(R)$ totally ordered, we were led to semi-invariant right chain rings. The valuation semigroup can then be described using Theorem 2. In many cases we actually have $H(R) \cong \widetilde{H}(R)$. The reason for this is the result we will prove in this section: Semi-invariant right chain rings with d.c.c. for prime ideals are right invariant. We recall that an ideal $P$ in $R$ is called completely prime if $a b$ in $P$ implies $a$ or $b$ in $P$ and $P$ is called prime if $a R b$ in $P$ implies $a$ or $b$ in $P$ where $a, b$ are elements in $R$. It follows from a result of Thierrin ([10]) that a prime ideal $P$ is completely prime if $a^{2}$ in $P$ implies $a$ in $P$.

Lemma 7. Every prime ideal $P$ in the semi-invariant ring $R$ is completely prime.

Proof. Assume $a^{2}$ in $P$ and $a$ not in $P$. Then there exists $t_{1}$ in $R$ with $a t_{1} a$ not in $P$ and $t_{2}$ in $R$ with $a t_{2}\left(a t_{1} a\right)$ not in $P$. We can assume $R \neq P$ and $a$ in $J$. Hence $a\left(t_{2} a t_{1}\right) a=a^{2} r$ for some $r$ in $R$ using (4) of Theorem 1. This contradiction proves the lemma.

The next result shows how to produce certain prime ideals.

LEMMA 8. Let $z$ be an element in $R$, a semi-invariant ring. Then $D=\cap z^{n} R$ is a prime ideal.

Proof. We can assume that $z$ is in $J$. Then $D$ is a right ideal and we will first show that $a^{2}$ in $D$ implies $a$ in $D$ for $a$ in $R$. Assume $a$ is not in $D$, then $a$ is in $J$ and $a j=z^{n}$ for some natural number $n$ and $j$ in $J$. But then $a j a j=a^{2} j^{\prime} j=z^{2 n}$ is not in $D$ contradicting $a^{2}$ in $D$. It remains to prove that $D$ is a left ideal. Let $x$ be in $D$ and $x=z^{n} q_{n}, q_{n}$ in $J$ follows. For $r$ in $R$ we get $r x r x=$ $r x r z^{n} q_{n}=z^{n} v q_{n}$ for some $v$ in $R$. This shows that $(r x)^{2}$ is in $D$ and hence $r x$ in $D$.

The next theorem will be proved in three steps, Lemmas 9-11.

THEOREM 3. A semi-invariant right chain ring with d.c.c. for ideals is right invariant.

Let $a$ be an element in the semi-invariant right chain ring $R$. By (5) Theorem 1 we have either $R a \subseteq a R$ or $J a \subseteq a J$. In the first 
case we are done and in the second we define a mapping $\phi$ from the set of prime ideals $P \neq R$ into itself by defining $P^{\phi}$ as the smallest prime ideal with $P a \subseteq a P^{\phi}$. We will show that either $J^{\phi}=J$ which implies $R a \leqq a R$ or $J^{\phi} \subset J$ and $\left\{J^{\phi^{n}}\right\}$ is a strictly decreasing chain of prime ideals of $R$.

Lemma 9. Let $J=J^{\phi}$ and $J=m R$, then $R a \leqq a R$.

Proof. We have $m a=a m^{k} v$ for some unit $v$ in $R$, some integer $k$, some generator $m$ of $J$, since as a right ideal $J^{\phi}=J$ using Lemma 8.

If $R a \nsubseteq a R$ there exists a unit $u$ in $R$ and an element $q$ in $J$ with $u a=a q$. Since $q$ is in $J$ and $u^{k+1} a=a q^{k+1}$ we obtain $q^{k+1} R \subset m^{k} R$ and we can assume $q R \subset m^{k} R$ and $q=m^{k} v t$ with $t$ in $J$. With $u s=m, m a=a m^{k} v, m a t=a m^{k} v t=a q=u a$ we obtain $s a t=a, s, t$ in $J$ and $a=0$ follows.

LeMma 10. Let $R$ be semi-invariant, $J$ not finitely generated as a right ideal and $0 \neq a$ an element in $R$ with $J a \subseteq a J^{\phi}, J^{\phi}=J$. Then $R a \subseteq a R$.

Proof. Assume $j \neq 0$ in $J$. We want to find $r, s$ in $J$ with $r a=a s$ and $s R \supseteqq j R$. Let $P=\cap j^{n} R$. By Lemma $8, P$ is a prime ideal and $P \subset J$. Since $J^{\phi}=J$ there exist elements $r_{1}, s_{1}$ in $J$ with $s_{1}$ not in $P$ such that $r_{1} a=a s_{1}$. Either $s_{1} R \supseteqq j R$ and we are done or there exists an $n$ with $j R \supset \cdots \supset j^{n-1} R \supset s_{1} R \supseteqq j^{n} R$. Hence $s_{1} q=j^{n}$ for some $q$ in $R$. We choose an element $z$ in $J$ with $r_{1}=z^{m} v$ with $v$ in $J$ and some $m>n$. This is possible, since $J$ is not finitely generated: Let $r_{1} R \subset x R \neq R$. We obtain $r_{1}=x y$ for $x, y$ in $J$. Choose $z_{1}$ in $J$ with $z_{1} R \supset x R$ and $z_{1} R \supset y R$ and $r_{1}=z_{1}^{2} u_{1}$ follows with $u_{1}$ in $J$. Repeating this process yields an element $z$ with $r_{1}=z^{m} v$, $z, v$ in $J, m>n$. Consider $z a=a z^{\prime}, z, z^{\prime}$ in $J$. We claim $z^{\prime} R \supseteqq j R$. Otherwise $j w=z^{\prime}$ for some $w$ in $J$. But $r_{1} a=z^{m} v a=a z^{\prime m} v^{\prime}=a s_{1}$ for some element $v^{\prime}$ in $J$ with $v a=a v^{\prime}$.

Hence $s_{1}=z^{\prime m} v^{\prime}=(j w)^{m} v^{\prime}=j^{m} b v^{\prime}$ for some element $b$ in $R$. This implies $j^{n}=s_{1} q=j^{m} b v^{\prime} q$, a contradiction, since $m>n$. We conclude that we have found an element $r=z, s=z^{\prime}$ with $s R \supseteqq j R$ and $r a=a s$ for the given element $j$ in $J$.

If $R a \nsubseteq a R$ there exist a unit $u$ in $R$ and an element $t$ in $J$ with $u a=a t$. By the above argument we have $s, r$ in $J$ with $r a=a s$ and $s R \supset t R$. Hence, $s v=t$ for some $v$ in $J$ and $r a v=a s v=$ $a t=u a$. We obtain $a=u^{-1} r a v=a k, k$ in $J$ and $a=0$, a contradiction. 
REMARK. Under the hypothesis of Lemma 10 we have proved that $J^{\phi}=J$ is even the smallest two-sided ideal $I$ satisfying $J a \leqq a I$.

LEMMA 11. Let $R$ be semi-invariant, a in $R$ with $J a \cong a J^{\phi}$ and $J^{\phi} \subset J$. Then $J^{\phi^{n+1}} \subset J^{\phi}$ for all $n$.

Proof. We will write $J^{(n)}$ instead of $J^{\phi^{n}}$. Then $J^{(n+1)} \subseteq J^{(n)}$ and we assume $n$ minimal with $J^{(n)}=J^{(n+1)}$. Let $r$ be in $J^{(n-1)} \backslash J^{(n)}$, $r a=a s$ with $s$ in $J^{(n)}$. Then there exists $a q$ in $J^{(n)}$ with $q a=a q^{\prime}$ and $q^{\prime} R \supset s^{k} R$ for some $k$, since otherwise $J^{(n+1)}=J^{(n)} \subseteq \cap s^{i} R \subset J^{(n)}$. After replacing $r$ by $r^{k}$ if $k>1$ we can assume that there is an $r$ in $J^{(n-1)} \backslash J^{(n)}$ with $r a=a s$ and an element $q$ in $J^{(n)}$ with $q a=a q^{\prime}$ and $q^{\prime} R \supset s R$. Hence $q^{\prime} t=s$ for some $t$ in $J$ and $r v=q$ for some $v$ in $J^{(n)}$. This yields $r a=a s=a q^{\prime} t=q a t=r v a t=r a v^{\prime} t$ with $v^{\prime}$ in $J$ and the contradiction $r a=0$ proves the lemma.

5. Examples, problems and comments. We begin with an example of a semi-invariant right chain ring $R$ such that $\widetilde{H}(R)$ contains $G$ where $G$ is a given totally ordered group.

ExAMPLE 1. For very totally ordered group $G$ there exists a semi-invariant right chain ring $R$ such that $\tilde{H}(R)$ contains $G$.

Let $K=\bigoplus_{i \in \boldsymbol{Z}} G_{i}$ where $G_{i} \simeq G$ for all $i \in Z . \quad K$ is an ordered group with the lexicographic ordering. Next, let $L=\left\{t^{n} k \mid n \in Z, k \in K\right\}$ with $t^{n} k_{1} \cdot t^{m} k_{2}=t^{n+m}\left(k_{1}^{(m)} k_{2}\right)$ be the ordered group where $k=\left(g_{i}\right)$ and $k^{(m)}=\left(g_{i}^{\prime}\right)$ with $g_{i}^{\prime}=g_{i+m}$. Further $t^{n} k_{1}>t^{m} k_{2}$ if and only if $n>m$ or $n=m$ and $k_{1}>k_{2}$ in $K$.

Let $H=\left\{t^{n} k \in L \mid t^{n} k \geqq e, k=\left(g_{i}\right)\right.$ with $n \geqq 0$ and $g_{i}=1_{G_{i}}$ for $i>0\}$. Then $H$ is a totally ordered semigroup with unit element and both cancellation laws. Further, $H$ is naturally ordered in the sense that $h_{1} \geqq h_{2}$ for $h_{i}$ in $H$ holds if and only if there exists an element $h \geqq e$ in $H$ with $h_{1}=h_{2} h$. Therefore it is possible to construct the generalized power series ring.

$$
\begin{gathered}
R^{\prime}=\left\{\alpha=\sum x_{h} a_{h} \mid h \in H, a_{h} \in R \text { and } T(\alpha)=\left\{h \mid a_{h} \neq 0\right\}\right. \\
\text { well ordered in } H\} .
\end{gathered}
$$

$R^{\prime}$ is a right invariant right chain ring with $\tilde{H}\left(R^{\prime}\right) \simeq H$ ([7]).

To the subsemigroup $M=\left\{t^{0}\left(g_{i}\right) \mid g_{i}=1_{G_{i}}\right.$ for $\left.i \neq 0\right\}$ there corresponds an $R^{\prime}$-convex subsemigroup in $\widetilde{H}\left(R^{\prime}\right)$ and a prime ideal $P$ in $R^{\prime}$. We put $R_{P}^{\prime}=R$. Since for $h$ in $M$ we have $h t=t h^{\prime}$ with $h^{\prime}$ not in $M$ unless $h=1$, we conclude that $\widetilde{H}(R) \simeq H M^{-1}=H \cup M^{-1}$. It follows that $G$ can be embedded into $\tilde{H}(R)$ where $R$ is a semiinvariant right chain ring. We observe that the right ideal $x_{t} R$ is 
not a left ideal and $R x_{t}$ is not a right ideal. On the other hand we know ([2]) that for every a in $a$ semi-invariant right and left chain ring either $a R$ or $R a$ is a two-sided ideal.

EXAMPLE 2. In our next example we construct a right chain ring $R$ such that $\tilde{H}(R)$ is not totally ordered, but that the subgroup $\widetilde{U}(R)=\{\tilde{u} \mid u$ in $U(R)\}$ of $\tilde{H}(R)$ is totally ordered with respect to the order as defined in $\widetilde{H}(R)$. This condition

\section{(U) $\widetilde{U}(R)$ is totally ordered}

is therefore weaker than the condition $\widetilde{H}(R)$ totally ordered and implies among other things that for a right chain ring $R$ with $(U)$, $a$ in $R$, there exists a unit $\varepsilon$ in $U(R)$ with $a \varepsilon$ in $\hat{R}$ (see Lemma 12 (ii) below). The basic idea of this construction has been used in [9], [2] and [6]: Let $R_{1}$ be a right and left chain ring, $D=Q\left(R_{1}\right)$ the division ring of quotients of $R_{1}, H$ a totally ordered semigroup with unit element that satisfies both cancellation laws. Further, let $h_{1} \geqq h_{2}$ hold for elements $h_{1}, h_{2}$ in $H$ if and only if $h_{1}=h_{2} h$ for some $h$ in $H$. Finally, let $\tau$ be a mapping from $H$ into the semigroup $M(D)$ of monomorphism from $D$ to $D$ with $\tau\left(h_{1} h_{2}\right)=\tau\left(h_{1}\right) \tau\left(h_{2}\right)$. One then can form the generalized power series ring $D\{\{H\}\}=\left\{\sum x_{h} d_{h}=\alpha \mid h\right.$ in $H, d_{h}$ in $D, T(\alpha)=\left\{h \mid d_{h} \neq 0\right\}$ well ordered in $\left.H\right\}$ where multiplication is defined by $x_{h_{1}} x_{h_{2}}=x_{h_{1} h_{2}}$ and $d x_{h}=x_{h} d^{\tau(h)}$. The subring $R$ of $D\{\{H\}\}$ consisting of those elements $\alpha$ with $d_{e}$ in $R_{1}$ is a right chain ring where $e$ is the unit element in $H$. It does not seem to be easy to determine $\widetilde{H}(R)$ in general.

To consider a special case let $F=Q(x, y)$, the field of rational functions in the two indeterminates $x$ and $y$ over the field $Q$ of rational numbers. Then $F$ contains $R_{1}=Q[x, y]_{(x)}$, a chain ring one obtains by localizing the polynomial ring $Q[x, y]$ at the prime ideal $(x)$. We form the skew power series ring $F[[t, \tau]]$, where $\tau$ is the automorphism of $F$ exchanging $x$ and $y$. Finally, $R$ consists of all those power series $\sum t^{i} f_{i}(x, y)$ with $f_{0}(x, y)$ in $R_{1}$. The principal right ideals of $R$ are of the form $t^{n} x^{m} R$ with $n=0,1,2, \cdots$ and $m$ in $Z$, but $m \geqq 0$ if $n=0$. The semigroup $\tilde{H}(R)=\left\{t^{n} x^{m} y^{k} \mid n=\right.$ $0,1,2, \cdots ; m, k$ in $\boldsymbol{Z}$ and $m \geqq 0$ if $n=0\}$. It is $t^{n_{1}} x^{m_{1}} y^{k_{1}}>t^{n_{2}} x^{m_{2}} y^{k_{2}}$ if $n_{1}>n_{2}$ or $n_{1}=n_{2}$ and $m_{1}>m_{2}$ with $k_{1} \geqq k_{2}$ or $n_{1}=n_{2}$, and $m_{1}=m_{2}$ and $k_{1}>k_{2}$. Finally, we have $\widetilde{U}(R)=\left\{\widetilde{y}^{k}, k \in Z\right\} \cong Z$ as ordered groups. Therefore, $\widetilde{H}(R)$ satisfies condition $(U)$, but is not totally ordered: $\tilde{x} \widetilde{y}^{-1}$ and $\tilde{1}$ for example cannot be compared.

We conclude this paper with some observation for right chain rings that satisfy condition $(U)$.

LEMma 12. Let $R$ be a ring satisfying condition $U$. 
(i) Let $a, b$ in $R$ with $a R=b R$. Then either $\widetilde{a} \leqq \tilde{b}$ or $\tilde{b}<\tilde{a}$.

(ii) For any $a$ in $R, R$ local, exists $x$ in $\hat{R}$ with $a R=x R$.

(iii) Let $R$ be a local ring and $a R \supset b R$. Then there exists for every $x$ with $x R=a R$ a $y$ in $R$ with $\tilde{x}<\tilde{y}$ and $y R=b R$. Similarly for every $y$ in $R$ with $y R=b R$ exists $x$ with $x R=a R$ and $\tilde{x}<\widetilde{y}$.

Proof. (i) is obvious, using condition $(U)$. Statement (ii) is correct if $a$ is a unit. We can therefore assume $a$ in $J, a$ not in $\hat{R}$. Hence $1+a$ is in $U(R) \backslash \hat{R}$ and $(1+a)(1+x)=(1+x)(1+a)=1$ for some $x$ in $R$. But $1+x$ and $x$ are in $\hat{R}$ and $a(1+x)=(1+x) a=$ $-x$ is in $\hat{R}$. Since $a R=x R$, (ii) follows.

To prove (iii) assume $b=x p$. Using (ii) there exists a unit $u$ in $R$ with $p u$ in $\hat{R}$ and $b u=x p u$ implies $\widetilde{x}<b \tilde{u}$. If $y=a p$ the second part of (iii) is correct for $p$ in $\hat{R}$. Otherwise we obtain with (ii): $(1+p)^{-1} p$ is in $\hat{R}, y=a(1+p)(1+p)^{-1} p$ and $x=a(1+p)$.

\section{Problems.}

(1) Describe all rings $R$ for which $\tilde{H}(R)$ satisfies $(U)$. (This class of rings contains all right invariant, in particular all commutative rings.)

(2) Which conditions characterize the semigroups $S$ with $S \cong$ $\tilde{H}(R), R$ a ring or additionally: $R$ a right chain ring.

(3) Find the class of rings $R$ with $\widetilde{H}(R)$ lattice ordered.

\section{REFERENCES}

1. R. T. Botto-Mura, H. H. Brungs and J. L. Fisher, Chain rings and valuation semigroups, Comm. Alg., 5 (1977), 1529-1547.

2. H. H. Brungs and G. Toerner, Chain rings and prime ideals, Arch. Math. (Basel), 27 (1976), 253-260.

3. P. M. Cohn, Free Rings and Their Relations, Academic Press, London, 1971.

4. P. Jaffard, Contribution a l'etude des groupes ordonnes, J. Mathes. Pures Appl., 32 (1953), 203-280.

5. W. Krull, Allgemeine Bewertungstheorie, J. Reine Angew. Math., 167 (1932), 160-196.

6. K. Mathiak, Bewertungen nicht kommutativer Koerper, J. Algebra, 48 (1977), 217-235.

7. B. H. Neumann, On ordered division rings, Trans. Amer. Math. Soc., 66 (1949), 202-252.

8. J. Ohm, Semi-valuations and groups of divisibility, Canad. J. Math., 21 (1969), 576-591.

9. W. Stephenson, Modules whose lattice of submodules is distributive, Proc. London Math. Soc., 28 (1974), 291-310.

10. G. Thierrin, Sur les idéaux complètements premiers d'un anneau quelconque, Acad. Roy. Belg. Bull. A. Sci., (5)43 (1957), 124-132.

Received September 2, 1979. The first author thanks the Technische Hochschule in Darmstadt for its hospitality during the first half of 1978 and the NRC for partial support. 
UNIVERSity of ALberta

Edmonton, Alberta, Canada

T6G 2G1

AND

Fachbereich Mathematik

Gesamthochschule Duisburg

41 DUISBURG

West Germany 



\section{PACIFIC JOURNAL OF MATHEMATICS}

EDITORS

DONALD BABBITT (Managing Editor)

University of California

Los Angeles, CA 90024

Hugo RossI

University of Utah

Salt Lake City, UT 84112

C. C. MOORE and ANDREW OGG

University of California

Berkeley, CA 94720

\section{J. DugundjI}

Department of Mathematics

University of Southern California

Los Angeles, CA 90007

R. FINN and J. MILGRAM

Stanford University

Stanford, CA 94305

\section{ASSOCIATE EDITORS}
R. ARENS
E. F. BECKENBACH
B. H. NEUManN
F. WOLF
K. YoSHIDA

\section{SUPPORTING INSTITUTIONS}

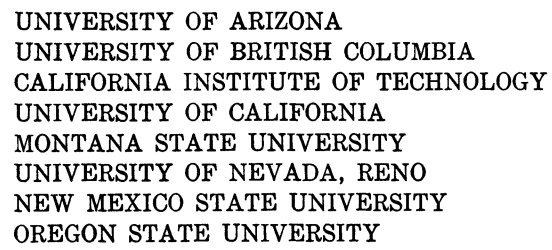

UNIVERSITY OF ARIZONA

UNIVERSITY OF BRITISH COLUMBIA

CALIFORNIA INSTITUTE OF TECHNOLOGY

UNIVERSITY OF CALIFORNIA

MONTANA STATE UNIVERSITY

UNIVERSITY OF NEVADA, RENO

NEW MEXICO STATE UNIVERSITY

OREGON STATE UNIVERSITY

\author{
UNIVERSITY OF OREGON \\ UNIVERSITY OF SOUTHERN CALIFORNIA \\ STANFORD UNIVERSITY \\ UNIVERSITY OF HAWAII \\ UNIVERSITY OF TOKYO \\ UNIVERSITY OF UTAH \\ WASHINGTON STATE UNIVERSITY \\ UNIVERSITY OF WASHINGTON
}

The Supporting Institutions listed above contribute to the cost of publication of this Journal, but they are not owners or publishers and have no responsibility for its content or policies.

Mathematical papers intended for publication in the Pacific Journal of Mathematics should be in typed form or offset-reproduced, (not dittoed), double spaced with large margins. Please do not use built up fractions in the text of the manuscript. However, you may use them in the displayed equations. Underline Greek letters in red, German in green, and script in blue. The first paragraph or two must be capable of being used separately as a synopsis of the entire paper. Please propose a heading for the odd numbered pages of less than 35 characters. Manuscripts, in triplicate, may be sent to any one of the editors. Please classify according to the scheme of Math. Reviews, Index to Vol. 39. Supply name and address of author to whom proofs should be sent. All other communications should be addressed to the managing editor, or Elaine Barth, University of California, Los Angeles, California, 90024.

50 reprints to each author are provided free for each article, only if page charges have been substantially paid. Additional copies may be obtained at cost in multiples of 50 .

The Pacific Journal of Mathematics is issued monthly as of January 1966. Regular subscription rate: $\$ 102.00$ a year (6 Vols., 12 issues). Special rate: $\$ 51.00$ a year to individual members of supporting institutions.

Subscriptions, orders for numbers issued in the last three calendar years, and changes of address shoud be sent to Pacific Journal of Mathematics, P.O. Box 969, Carmel Valley, CA 93924, U.S.A. Old back numbers obtainable from Kraus Periodicals Co., Route 100, Millwood, NY 10546.

\section{PUBLISHED BY PACIFIC JOURNAL OF MATHEMATICS, A NON-PROFIT CORPORATION}

Printed at Kokusai Bunken Insatsusha (International Academic Printing Co., Ltd.). 8-8, 3-chome, Takadanobaba, Shinjuku-ku, Tokyo 160, Japan. 


\section{Pacific Journal of Mathematics}

\section{Vol. 97, No. $2 \quad$ February, 1981}

Patrick Robert Ahern and N. V. Rao, A note on real orthogonal measures . . . . . 249

Kouhei Asano and Katsuyuki Yoshikawa, On polynomial invariants of fibered

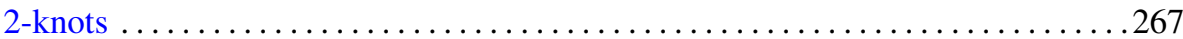

Charles A. Asmuth and Joe Repka, Tensor products for $S L_{2}(\mathscr{K})$. I.

Complementary series and the special representation

Gary Francis Birkenmeier, Baer rings and quasicontinuous rings have a

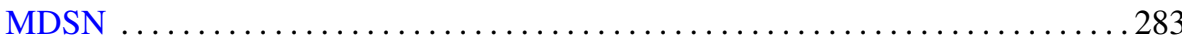

Hans-Heinrich Brungs and Günter Törner, Right chain rings and the generalized

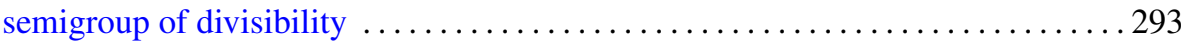

Jia-Arng Chao and Svante Janson, A note on $H^{1} q$-martingales . . . . . . . . . 307

Joseph Eugene Collison, An analogue of Kolmogorov's inequality for a class of

additive arithmetic functions

Frank Rimi DeMeyer, An action of the automorphism group of a commutative ring on its Brauer group

H. P. Dikshit and Anil Kumar, Determination of bounds similar to the Lebesgue

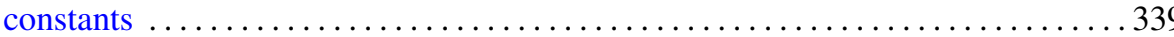

Eric Karel van Douwen, The number of subcontinua of the remainder of the plane

D. W. Dubois, Second note on Artin's solution of Hilbert's 17th problem. Order spaces

Daniel Evans Flath, A comparison of the automorphic representations of GL(3) and its twisted forms

Frederick Michael Goodman, Translation invariant closed $*$ derivations

Richard Grassl, Polynomials in denumerable indeterminates

K. F. Lai, Orders of finite algebraic groups

George Kempf, Torsion divisors on algebraic curves

Arun Kumar and D. P. Sahu, Absolute convergence fields of some triangular matrix methods

Elias Saab, On measurable projections in Banach spaces

Chao-Liang Shen, Automorphisms of dimension groups and the construction of AF algebras

Barry Simon, Pointwise domination of matrices and comparison of $\Phi_{p}$ norms

Chi-Lin Yen, A minimax inequality and its applications to variational inequalities

Stephen D. Cohen, Corrections to: "The Galois group of a polynomial with two indeterminate coefficients"

Phillip Schultz, Correction to: "The typeset and cotypeset of a rank 2 abelian group"

Pavel G. Todorov, Correction to: "New explicit formulas for the $n$th derivative of composite functions"

Douglas S. Bridges, Correction to: "On the isolation of zeroes of an analytic function" 